\section{The core and conserved role of MAL is homeostatic regulation of actin levels}

\author{
Lara Salvany, Julius Muller, Ernesto Guccione, \\ and Pernille Rørth ${ }^{\mathbf{1}}$ \\ Institute of Molecular and Cell Biology, Singapore 138673, \\ Singapore
}

The transcription cofactor MAL is regulated by free actin levels and thus by actin dynamics. MAL, together with its DNA-binding partner, SRF, is required for invasive cell migration and in experimental metastasis. Although MAL/SRF has many targets, we provide genetic evidence in both Drosophila and human cellular models that actin is the key target that must be regulated by MAL/SRF for invasive cell migration. By regulating MAL/SRF activity, actin protein feeds back on production of actin mRNA to ensure sufficient supply of actin. This constitutes a dedicated homeostatic feedback system that provides a foundation for cellular actin dynamics.

Supplemental material is available for this article.

Received January 8, 2014; revised version accepted April 16, 2014.

The transcription cofactor MAL is regulated by cellular actin dynamics and confers this regulation on the activity of its DNA-binding partner, SRF (Sotiropoulos et al. 1999; Posern et al. 2002; Miralles et al. 2003). Free G-actin directly binds to MAL via RPEL motifs at the $\mathrm{N}$ terminus of MAL and negatively regulates its activity. This regulation is conserved from mammals to insects (Somogyi and Rørth 2004). In the physiological context of the animal, the function of MAL and related proteins (MRTFA and MRTF-B in mammals and mal-d/mrtf in Drosophila) appears conserved as well, related to active changes in the cytoskeleton. For example, initiation of invasive cell migration is essentially abolished in the absence of MAL or SRF in border cell migration in the Drosophila ovary (Somogyi and Rørth 2004) or in mouse bipolar neurons exiting the subventricular zone of the brain (Pinheiro et al. 2011) and for cancer cells in threedimensional (3D) invasion assays and experimental metastasis (Medjkane et al. 2009).

Actin is a very abundant and exquisitely conserved protein in eukaryotic cells. Cycling of actin between G-actin and F-actin pools is controlled by a vast array of regulators, which have been the focus of considerable attention (Pollard and Borisy 2003). Actin protein synthesis is also a regulated process (Bershadsky et al. 1995; Lyubimova et al. 1999; Huttelmaier et al. 2005). Actin

[Keywords: actin synthesis; invasive cell migration; transcriptional regulation]

${ }^{1}$ Corresponding author

E-mail prorth@imcb.a-star.edu.sg

Article is online at http://www.genesdev.org/cgi/doi/10.1101/gad.237743.114.
mRNA localization and localized protein synthesis are important for cell migration and axonal growth and guidance (Huttelmaier et al. 2005; Yao et al. 2006). Here, we present evidence that regulation of actin gene transcription is itself a key regulatory step in the control of invasive cell migration. Using a genome-wide approach, we identified Actin5C as a major target of the MAL/SRF transcription factor complex. Loss or reduction of MAL activity impairs invasive migration in Drosophila and human cancer cell models. We found that restoring actin expression can be sufficient to replace the requirement for MAL to support invasive migration in these models. Thus, actin and MAL form a conserved homeostatic feedback system to ensure that actin levels are appropriate to support the actin dynamics required for complex cell behavior.

\section{Results and Discussion}

To understand why MAL is essential for invasive migration and whether the apparent similarity of its role in different organisms reflects a conserved molecular mechanism, we set out to identify Drosophila MAL target genes at the genome level. We used a combination of chromatin immunoprecipitation (ChIP) and gene expression analysis and focused on MAL, as SRF has functions independent of MAL/mrtf in mammals and flies (Whitmarsh et al. 1995; Montagne et al. 1996). To perform analysis in the relevant tissue context, we used a mutant in the single Drosophila mal-d gene that abolishes expression in the ovary (mal- $d^{\Delta 7}$ ) (Somogyi and Rørth 2004). This specifically blocked invasive migration by border cells and caused overall ovary growth defects (Fig. 1A,B; Somogyi and Rørth 2004). Ubiquitous expression of a GFP-tagged version of Mal-d completely rescued the mal-d mutant phenotypes (Fig. 1C,D), showing that the fusion protein provides normal Mal-d function. The MAL-GFP transgene also allowed efficient identification of MAL-GFP-bound regions in the Drosophila genome by immunoprecipitation with GFP (Fig. 1E; Supplemental Table S1). Key MAL-GFP-bound regions were confirmed in independent samples, and their recovery was dependent on the presence of the transgene (Supplemental Fig. S1). In parallel, genome-wide expression analysis of wild-type versus mal- $d$ mutant ovaries identified genes whose expression was dependent on MAL (Fig. 1E; Supplemental Table S2). These two complementary data sets allowed genome-wide identification of MAL target genes. Enriched genome annotation (gene ontology [GO]) categories for both data sets are shown in Supplemental Figure S2.

There were two key findings from this genome-wide analysis. First, only a small number of genes qualified as direct MAL targets, with MAL-GFP bound to the regulatory region and a significant decrease of mRNA expression in the mutant: the cytoplasmic Actin5C gene and five other genes, most encoding heat-shock proteins (Fig. 1E). A few additional genes encoding cytoskeletal proteins or

(c) 2014 Salvany et al. This article is distributed exclusively by Cold Spring Harbor Laboratory Press for the first six months after the fullissue publication date (see http://genesdev.cshlp.org/site/misc/terms.xhtml). After six months, it is available under a Creative Commons License (Attribution-NonCommercial 4.0 International), as described at http:// creativecommons.org/licenses/by-nc/4.0/. 

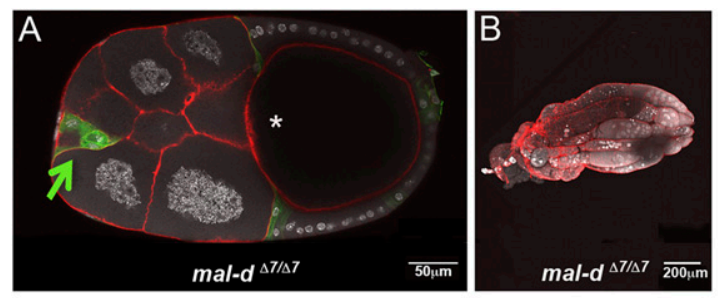

$\mathrm{E}$
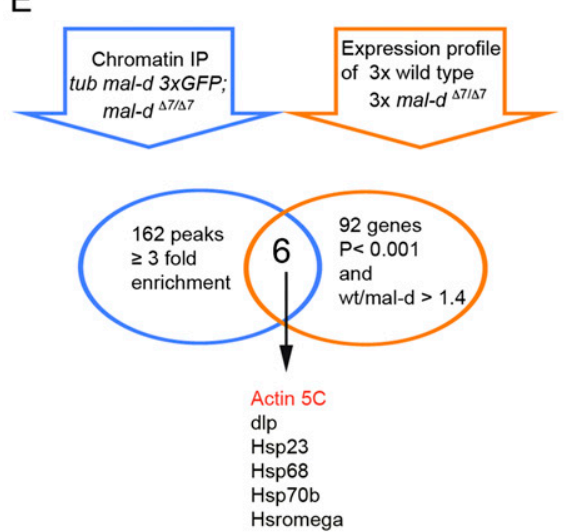

$\mathrm{F}$

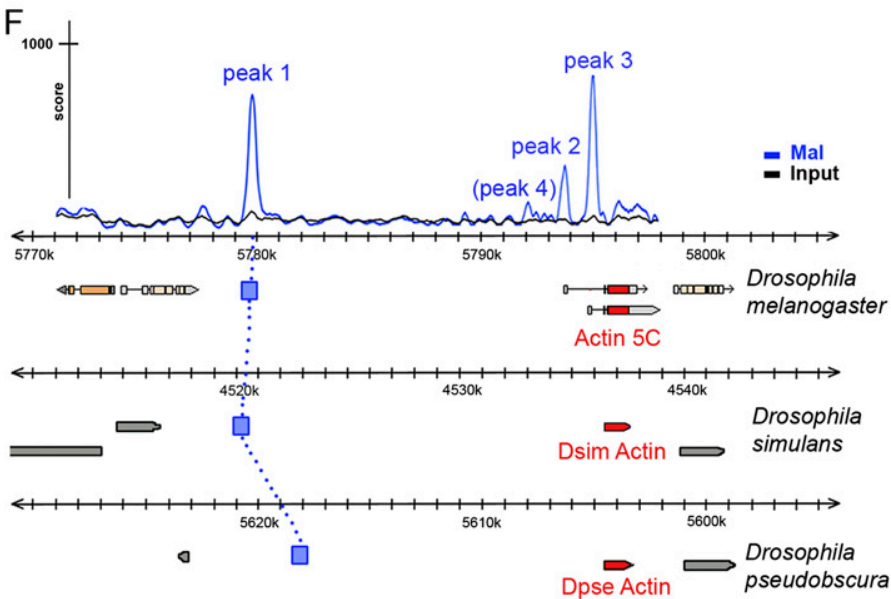

Figure 1. Identification of direct target genes for mal- $d$ in Drosophila. (A) Stage 10 egg chamber from a mal- $d^{\Delta 7}$ homozygous female. The border cell cluster is labeled by GFP and indicated by a green arrow. Anterior is to the left in this and all other images. Border cells normally migrate from the anterior end and to the oocyte (white asterisk in $A$ and $C$ ) by stage 10 (as in $C$ ), so migration here is severely defective. DAPI (nuclei) is in gray, and phalloidin (F-actin) is in red. (B) Ovary from a mal- $d^{\Delta 7}$ homozygous female stained with DAPI (gray) and phalloidin (red). (C) Stage 10 egg chamber and ovary (shown in $D$ ) from tub mal-d3xGFP; mal- $d^{\Delta{ }^{7}}$ homozygous females. Ubiquitous expression of mal $d 3 x G F P$ rescues border cell migration (green arrow) and ovary defects (stained as in $A$ and $B$ ). (E) Diagram of strategy to identify direct targets of mal-d. ChIP on tub mal$d 3 x G F P ;$ mal- $d^{\Delta 7}$ ovaries identified 168 enriched peaks. Expression profiling of ovaries identified 98 genes with a significant decrease in expression in mal- $d^{\Delta 7}$ homozygous females compared with wild type $\left(O R^{+}\right)$. The overlap is the six listed genes. $(F$, top $)$ Binding profile of Mal-dGFP to the Actin5C genomic region (blue) represented by score (number of reads per peak). Actin5C mRNA and ORF are indicated below (red). Peaks 2 and 3 (and peak 4) are in the promoter region, and peak 1 is $10 \mathrm{~kb}$ upstream. (Below) Aligned genomic regions from other Drosophilids (Drosophila simulans and Drosphi; a pseudoobscura) (same scale; data from FlyBase). The actin genes are highly conserved, the overall locus organization is similar, and the upstream peak 1 is conserved (blue boxes).

regulators were identified as potential targets in the MAL-GFP-bound set (Supplemental Table S1). Second, three of the four most enriched MAL-bound regions in the whole genome were associated with Actin5C (Fig. 1F; Supplemental Table S1). The MAL-bound regions were conserved in other species, suggesting functional importance, and in each case, these sites bracketed gene-free upstream regions of $\sim 10 \mathrm{~kb}$ (Fig. 1F). The latter is noteworthy because the Drosophila genome is dense, with most "housekeeping genes" closely spaced, and large regulatory regions generally confined to developmental regulators. These findings focused our attention on Actin5C.

The gene expression arrays indicated a modest decrease of Actin5C mRNA levels in the mal-d mutant (Supplemental Table S2). Quantitative RT-PCR of carefully matched ovary samples showed a twofold decrease of mature Actin5C mRNA and a threefold to fourfold decrease of primary transcript in mal-d mutants (Fig. 2A,B), with no change in the closely related, but less highly expressed, Actin42A gene (Wagner et al. 2002). In FACSsorted migratory cells, including border cells, Actin5C levels were fourfold reduced (Supplemental Fig. S3). Analysis of the Actin5C promoter and upstream region in luciferase reporter assays showed robust promoter activity (2,3-luc and 1,2,3-luc in Fig. 2C) and 200-fold to 600-fold up-regulation by coexpression of SRF and activated MAL (mal-d- $\Delta$ N) (Fig. 2D; Somogyi and Rørth
2004). Conversely, knockdown of MAL or SRF by RNAi gave 50-fold to 100-fold reduction in basal Actin5C expression (Fig. 2E). The Actin5C regulatory region also conferred responsiveness to drugs affecting actin dynamics, specifically induction by Cytochalasin $\mathrm{D}$ and inhibition by Latrunculin B (Fig. 2F), as observed for mammalian MAL/SRF-regulated genes. This type of reporter assay generally reveals regulatory potential at the transcriptional level. The large magnitude of regulation of the Actin5C promoter/enhancer region by MAL/SRF is consistent with the abundant binding of MAL to this region (Fig. 1F). In vivo, compensatory mechanisms may contribute to sustaining Actin5C mRNA levels upon loss of MAL activity. Thus, MAL and actin dynamics have the potential to regulate Actin5C transcription over a large dynamic range.

The genomic data raised the possibility that Actin5C might not be just one of many cytoskeletal target genes for MAL regulation but the key target gene. If a transcription factor has one key target gene in vivo, re-expression of this gene should replace the need for the transcription factor. In genetic terms, expression of the target gene should rescue the phenotype of complete loss of function for the transcription factor in specific cells.

To investigate this hypothesis functionally, we turned to the severe defect in invasive migration observed in mal-d mutant border cells (Fig. 3A). Actin5C is the major cytoplasmic actin gene, and, as expected, mutating it 

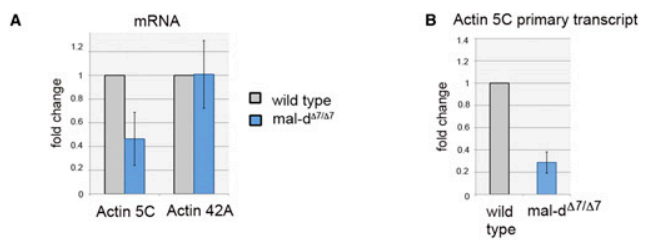

C

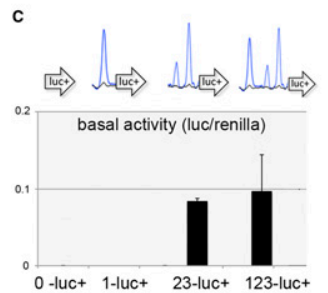

D

750 fold induction with SRF+ mal-d $\mathrm{d}^{\mathrm{N}}$

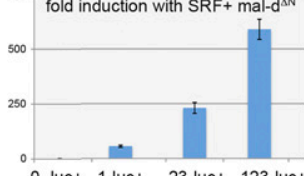

0 -luct 1-luct 23-luct 123-luct
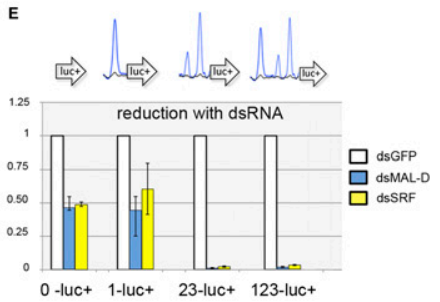

$\mathbf{F}$

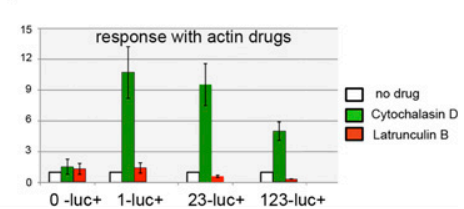

Figure 2. Analysis of Actin5C transcriptional regulation by mal-d. $(A, B)$ Quantification of transcript levels in wild-type and mal- $d^{\Delta 7}$ mutant ovaries by quantitative PCR. Mature (mRNA) transcript of Actin5C and Actin42A $(A)$ and Act5C primary transcript (primer in intron sequences) $(B)$; mean $\pm \mathrm{SD}$ from three biological replicates. $(C-F)$ Reporter assays from Schneider S2 cells transfected with firefly luciferase constructs containing genomic DNA from the Actin5C locus as shown schematically above the graphs: peak $1\left(1-\mathrm{luc}^{+}\right)$, peaks 2 and 3 $\left(23-\right.$ luc $\left.^{+}\right)$, peaks 1,2 , and $3\left(123-\right.$ luc $\left.^{+}\right)$, or empty vector $\left(0-u_{c}{ }^{+}\right)$. The ratio of firefly to control Renilla luciferase activity is shown as the mean \pm SD from three independent experiments. In $D$, cells were cotransfected with pRm-SRF and pRm-mal-d- $\Delta \mathrm{N}$ (activated mal-d), and the data were normalized to parallel transfection with empty pRm vector. In $E$, cells were treated with dsRNA against mal-d (dsMAL-D), srf (dsSRF), or GFP (dsGFP), and the data were normalized to the control (dsGFP) sample for each construct. $(F)$ Effect of actin polymerization drugs on Actin5C promoter/enhancer activity: Cells were incubated with $5 \mu \mathrm{M}$ Cytochalasin D or $500 \mathrm{nM}$ Latrunculin B for $7 \mathrm{~h}$ before harvest. Each construct is normalized to a parallel experiment without drug.

perturbs border cell migration (Geisbrecht and Montell 2004). To determine whether Actin5C was the sole required target gene of MAL, we tested whether re-expression of Actin5C in cells that appear to be null for mal-d ( all $^{S 2}$ ) (Somogyi and Rørth 2004) could restore invasive migration. We used fly strains in which the Actin5C gene has a Gal4-responsive transposon, an "EP element," in the promoter region. Surprisingly, migration was indeed restored to normal when Actin5C was activated by Gal4 in mal-d mutant cells (Fig. 3A-C). Thus, as long as the Actin5C gene is induced at an adequate level (see Supplemental Fig. S3), border cells do not need MAL to invade and migrate.

In fully mutant ovaries (mal- $d^{\Delta 7}$ homozygous females), restricted expression of Actin5C in terminally differentiated outer border cells using slbo-Gal4 provided significant but less efficient rescue of migration (Fig. 3D) despite normal expression levels (Supplemental Fig. S3). This suggested that MAL also acts in other cells, consistent with the general oogenesis phenotype (Fig. 1B). The ORF of Actin5C in a Gal4-reponsive transgene (UAS-Actin-ORF) also provided some rescue of migration, whereas a construct with stop codons present (Stop) did not (Fig. 3D). This confirmed that actin protein expression was re-

sponsible for the activity of the Actin5C locus in border cells.

To test whether this finding extended to other tissues, we analyzed another mal-d mutant phenotype, bent bristles. Bristles are "hairs" organized by actinrich structures (Tilney et al. 1996) and are characteristically defective in mal-d mutants (Somogyi and Rørth 2004). This effect of MAL deficiency was also restored to normal by ectopic expression of the Actin5C, accomplished by placing Actin5C cDNA under the control of a heat-shock promoter and rearing at $29^{\circ} \mathrm{C}$ (Fig. $3 \mathrm{E}, \mathrm{F})$. Thus, the requirement for MAL in these contexts reflects a specific need for MAL-driven induction of Actin5C expression. Regulation of other targets, direct or indirect, is not required. These findings indicate that the primary role of Drosophila MAL is to regulate actin levels in response to free actin fluctuations, a homeostatic feedback regulation.

We next asked whether this role of MAL as regulator of actin homeostasis is conserved in mammalian cells. The cytoplasmic $\beta$-actin and $\gamma$-actin genes are regulated by MAL/ SRF in mammalian cells (Miano et al. 2007; Luxenburg et al. 2011). As for Drosophila Actin5C, $\beta$-actin is essential for embryonic development and proper cell migration, whereas the related $\gamma$-actin can be compensated for (Bunnell et al. 2011). However, many other genes are more dramatically regulated by SRF and mrtfs, and some of these are important for cell migration and related functions (Medjkane et al. 2009). It is therefore assumed that MAL and SRF exert their function by regulating a battery of cytoskeletal genes. For SRF, the number of direct target genes is estimated at 200-300 (Cooper et al. 2007; Miano et al. 2007). For MAL and MRTFs, this is less clear, as systematic ChIP analysis is missing. Based on our findings in the fly model, we decided to test the hypothesis that a single target gene ( $\beta$-actin) could be the key effector of mammalian MAL and that its expression could replace the need for MAL-driven gene regulation in an assay of invasive cell migration.

The requirement for MAL is most pronounced in cases of active cell shape change and cytoskeletal challenge, such as tissue or matrix invasion (Somogyi and Rørth 2004; Medjkane et al. 2009; Pinheiro et al. 2011). We therefore looked for a simple cellular assay that could test the ability of cells to invade into a confined environment. Migration under agarose provides mechanical resistance to movement and has been used to study migration of eukaryotic cells in a constrained space (Nelson et al. 1975; Laevsky and Knecht 2001, 2003). The assay can easily be adapted to human tumor cells such as MDA-MB-231 breast cancer cells (Fig. 4A; Wiggins and Rappoport 2010). MAL activity in these cells is provided by mrtf-a and mrtf-b, and reducing their expression severely reduces invasion in an organotypic assay and in experimental metastasis in mice (Medjkane et al. 2009). Simultaneous knockdown of mrtf-a and mrtf-b in MDA-MB-231 cells by siRNA reduced $\beta$-actin transcript levels (Fig. 4B). It also produced severe attenuation of migration under agarose (Fig. 4C), confirming that this assay interrogates MALdependent cell movement.

To assess the importance of cytoplasmic actin downstream from MAL, we derived stable cell lines from MDA-MB-231 that allowed the ORF of human $\beta$-actin, or an $\mathrm{N}$-terminally Flag-tagged version of $\beta$-actin, to be induced from the strong CMV promoter by the TET-ON 

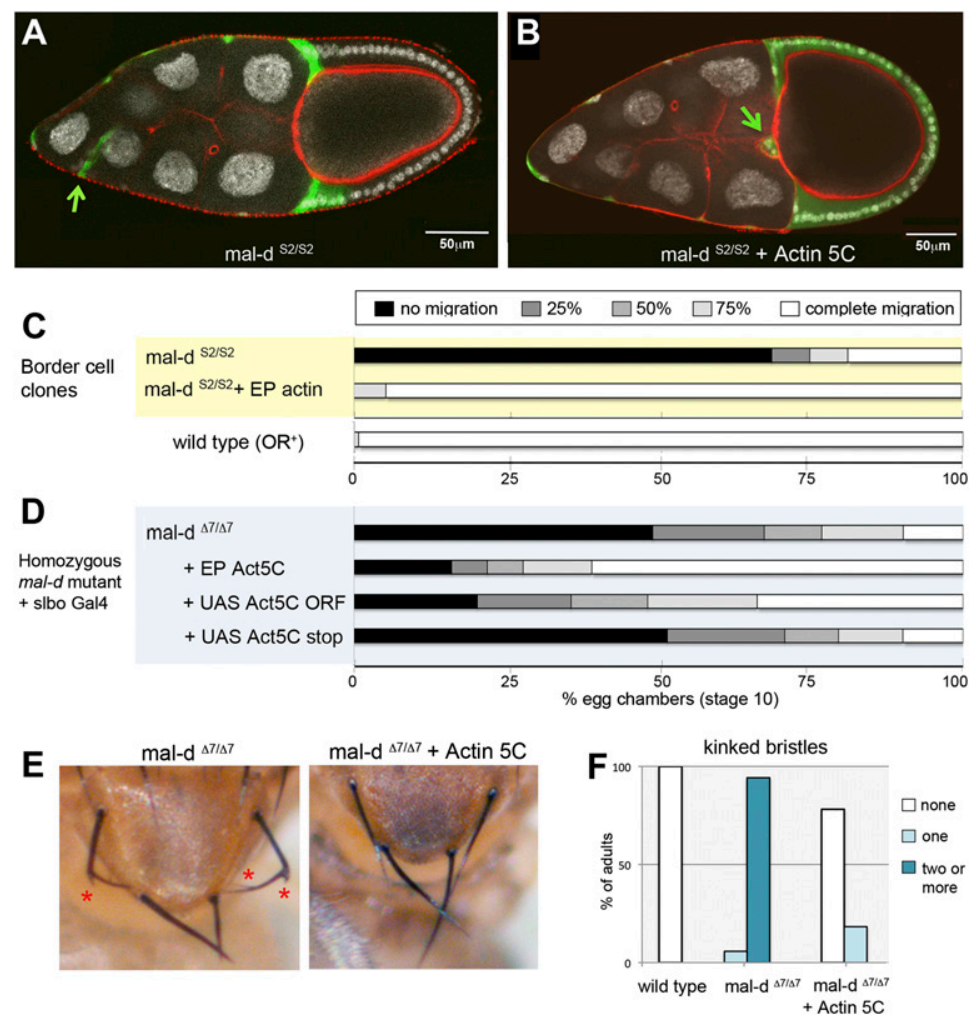

Figure 3. Expression of Actin5C rescues mal-d mutant phenotypes in Drosophila. (A) Border cells lacking mal-d fail to migrate. Stage 10 egg chamber of genotype hsFLP; tubulin Gal4, UAS GFP; mal-d ${ }^{\text {S2 }}$,FRT80/Gal80,FRT80 (MARCM system). Homozygous mutant cells are marked by GFP, and border cells are indicated by a green arrow. Nuclei are stained with DAPI (gray), and F-actin is stained with phalloidin (red). (B) Border cells lacking mal-d but re-expressing Actin5C migrate normally. Stage 10 egg chamber of genotype EP actin/hsFLP; tubulin-Gal4, UASGFP; mal-d ${ }^{S 2}$ FRT80/ Gal80,FRT80; markers are as in $A .(C, D)$ Quantification of border cell migration scored in stage 10 egg chambers. $(C)$ mal- $d$-null mutant border cells without or with expression of Actin5C as in $A$ and $B ; n=16$ and 38, respectively. Wild type is shown below $(98 \%$ complete migration at stage $10 ; n=$ 721). (D) Females homozygous mutant for mal- $d^{\Delta 7}$ with expression of the indicated EP insertions or UAS transgenes by slbo Gal4. Genotypes are slbo-Gal4, mal$d^{\Delta 7} / \mathrm{mal}^{-} d^{\Delta 7}(n=1323)$, EP-Act5C; $/+$; slbo-Gal4, mal- $d^{\Delta 7} / \mathrm{mal}^{-} d^{\Delta 7}(n=1255)$, UAS-Act5C-ORF/+; slbo-Gal4, mal-d $d^{\Delta 7} / \mathrm{mal}^{-d^{\Delta 7}}(n=2378)$, and UAS-Act5CStop/+; slbo-Gal4, mal- $d^{\Delta 7} / \mathrm{mal}^{-d^{\Delta 7}}(n=929)$. Act5C-Stop is as Act5C-ORF but with two early stop codons. (E, left panel) Scutellum from a mal-d $d^{\Delta 7}$ homozygous adult. Kinked bristles are marked by red asterisks. (Right panel) Scutellum from a HS-Act5C, mal- $d^{\Delta 7}$ homozygous adult. Actin5C cDNA under the control of the heat-shock promoter rescues the bristle defect. Flies were kept at $29^{\circ} \mathrm{C}$ from third larval instar. $(F)$ Quantification of kinked bristles in scutellum in wild type $\left(\mathrm{OR}^{+}\right)(n=$ $144)$, mal- $^{\Delta 7}(n=89)$, or HS-Act5C, mal- $d^{\Delta 7}(n=93)$.

system. These cell lines showed some baseline expression of the transgenes and six-fold to 10-fold induction upon Tet addition (Supplemental Fig. S4A). Under agarose, migration was similar to the parental cell line (Fig. 4D,E) and not changed by Tet addition (Supplemental Fig. S4B). Knockdown of mrtf-a and mrtf-b largely attenuated migration under agarose. Remarkably, ectopic induction of $\beta$-actin or Flag- $\beta$-actin in the mrtf-a/b-depleted cells rescued migration to control levels (Fig. 4D,E). This indicates that human cancer cells require MAL activity to perform invasive migration for the exact same reason that Drosophila border cells do: to regulate cytoplasmic actin gene expression. Regulation of any other potential MAL target genes may not be required for this cell behavior. These experiments rely on siRNA-mediated reduction of mrtf activity; thus, it remains possible that other target genes are important but require only low levels of mrtf activity.

Elegant experiments have shown how activity of SRF and its transcriptional cofactor, MAL, is regulated by the level of free actin and thereby by the dynamics of the actin cytoskeleton (Sotiropoulos et al. 1999; Posern et al. 2002; Miralles et al. 2003). Here we show that the key role of MAL is to regulate the expression level of cytoplasmic actin. We suggest that this simple feedback system allows cells to produce sufficient free actin to meet the needs of their changing cellular cytoskeletal dynamics. The extensive MAL-decorated regulatory region displayed by Actin5C may serve to tune actin gene expression sensitively and accurately. We provide evidence that cytoplasmic actin is the sole critical target gene for invasive migration in Drosophila and possibly also in human cells. We therefore propose that this regulation is the ancestral function of the MAL/SRF complex in animal cells. Additional target genes for MAL have been acquired in different species and are likely to contribute to cell fitness. Examples include other cytoskeletal proteins (Medjkane et al. 2009) as well as genes encoding heat-shock proteins (this study), some of which may interact with actin (Lavoie et al. 1993; Mounier and Arrigo 2002). While some of these genes appear more dramatically regulated by MAL when considering relative mRNA levels, actin may be the "most regulated" gene if considering the number of transcripts induced. In any case, identification of actin as the core, essential target of MAL reveals the core of this transcription regulatory "network" to be a simple and logical feedback system.

Why is the MAL-driven regulation of actin particularly critical for invasively migrating cells? The stimuli inducing a robust F-actin-based cytoskeleton when initiating migration into constrained space is likely to convert free G-actin into F-actinrich structures. Maintaining the appropriate free actin pool for further cytoskeletal buildup or for other cellular functions then requires new production of actin. Other dramatic shape changes, such as cells rounding up in the stratified epidermal layer of the skin, may induce similar sudden actin pool depletion and therefore require MAL and SRF (Luxenburg et al. 2011). We previously suggested that MAL forms part of a mechanical feedback system for invasive cells (Somogyi and Rørth 2004) whereby mechanical tension induces MAL activity in order to make "robust" cells. Related concepts of tension-driven responsiveness have recently been indicated for mammalian MAL/SRF function as well (Connelly et al. 2010; McGee et al. 2011). The mechanical feedback logic is fully compatible with the simple molecular feedback system presented here. It can be regarded as an alternate point of perturbation impinging on actin homeostasis; namely, stretching or stressing cells to provoke a biomechanical cytoskeletal response. Robust feedback systems such as this one driven by MAL are likely to be well conserved through animal evolution, when the target is a crucial one. The ability of cells to 
A

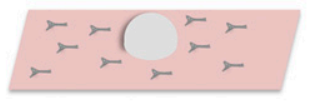

$\underline{\text { C }}$

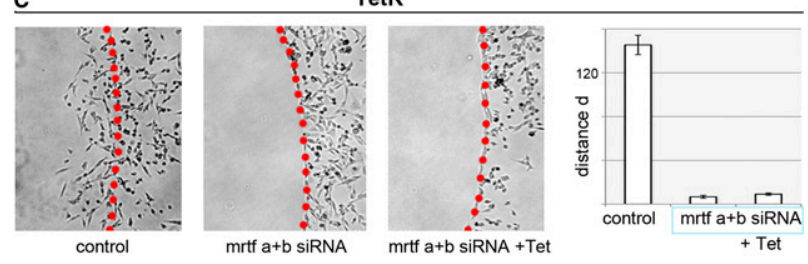

$\underline{\text { D }}$

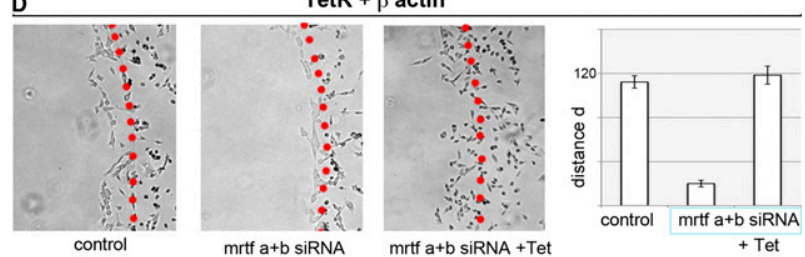

mrtf a+b siRNA +Tet
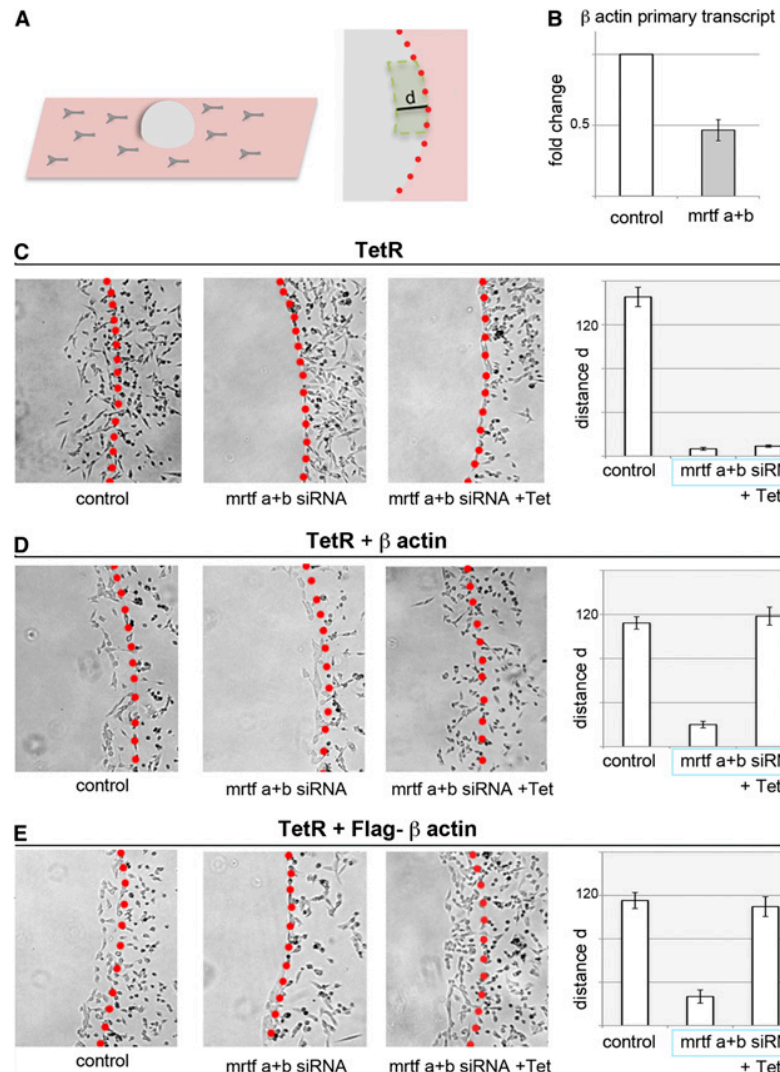

TetR + Flag- $\beta$ actin
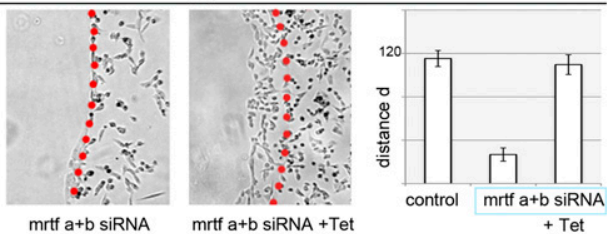

Figure 4. Re-expression of $\beta$-actin in human breast cancer cells depleted for mrtf-a and mrtf-b rescues invasive migration defect. $(A)$ Diagram of agarose spot assay. Cells were seeded in wells (pink surface) with drops of solidified agarose (gray sphere) and invaded along the bottom surface under the agarose. Pictures were taken along the edge (see $C-E$; the edge is indicated by a line of red dots), and representative regions were measured. The distance, $d$, is the extent of invasion under agarose from the drop edge for a section of the cell "sheet." $(B) \beta$-Actin primary transcript levels in mrtf-a + bdepleted MDA-MB 231 cells and control cells; mean \pm SD from three experiments. $(C-E)$ Representative images and quantification of under agarose invasion assay for the indicated MDA-MB-231 cell lines and treatments. (C) TetR: MDA-MB-231 cells expressing tetracycline repressor (parental line). The derivative lines below also express human $\beta$-actin (TetR $+\beta$-actin) $(D)$ or a Flag-tagged version (TetR + Flag- $\beta$-actin) $(E)$ from a Tet-inducible vector. Panels from left to right: cells treated with negative control siRNA (control), cells treated with siRNA for both mrtf-a and mrtfb (mrtf-a + b siRNA), and cells treated with siRNA for both mrtf$\mathrm{a}$ and mrtf-b and tetracycline added (mrtf-a + b siRNA + Tet) Quantification is shown at the right as mean \pm SEM of 12 measurements from three independent experiments.

occasionally migrate and invade is a characteristic of animal systems, and the dynamic actin cytoskeleton is central to this behavior.

\section{Materials and methods}

\section{ChIP}

ChIP was performed on whole ovaries (500 ovaries per immunoprecipitation) from wild-type $\left(\mathrm{OR}^{+}\right)$or tubmal3xGFP;mal- $d^{D 7} / \mathrm{mal}^{-} d^{D 7}$ females and prepared as previously described (Negre et al. 2006). For MAL-GFP immunoprecipitation, $60 \mathrm{~mL}$ of GFP beads (GFP-Trap ChromoTek) was used For controls, an equivalent volume of protein-A beads was used, or immunoprecipitation with GFP beads was performed on $\mathrm{OR}^{+}$extract. The DNA was PCR-amplified using Applied Biosystems 7500 Fast equipment and software.

\section{ChIP sequencing}

Library preparation for deep sequencing of 15 ng of MAL-GFP immunoprecipitation DNA and $15 \mathrm{ng}$ of input DNA from the tubmal3xGFP;mal$d^{D 7}$ sample was performed according to Illumina recommendations. For immunoprecipitation and input control samples, 76 million and 83 million 35-base-pair (bp) reads, respectively, were uniquely mapped by the CASAVA pipeline to the $\mathrm{dm} 3$ version of the Drosophila melanogaster genome. MACS version 2.09 (Zhang et al. 2008) was used to detect peaks, allowing for a maximum of six reads at the same position. A peak was considered significant if the multiple-testing-corrected $P$-value was $<0.001$ and the enrichment was greater than threefold over input.

\section{Expression profiling by microarrays}

Frozen homogenized samples were prepared from three sets of each 75 control $\left(\mathrm{OR}^{+}\right)$or mal- $d^{D 7} / \mathrm{mal}_{-} d^{D 7}$ mutant ovaries aged $18 \mathrm{~h}$. These triplicate samples were converted to cDNA probes and compared by hybridization to Flychip FL003 microarrays by the Cambridge Microarray Facility. The expression data from Flychip FL003 arrays (Gene Expression Omnibus platform accession no. GPL8244) were subjected to variance stabilization normalization (VSN) (Huber et al. 2002). Only transcripts with a Benjamini and Hochberg multiple-testing-adjusted $P$-value of $<0.001$ and absolute change $>1.4$-fold ( $40 \%$ higher in wild type than in the mal-d mutant) were considered significantly differentially expressed.

\section{RNA extraction and quantitative PCR analysis}

Ovary samples from females $0-4 \mathrm{~h}$ after eclosion were used for best quantitative comparison, three samples of each 30 ovaries per genotype. RNA was prepared using RNeasy minikit (Qiagen), and $1 \mu \mathrm{g}$ of total RNA was used for reverse transcription and first strand cDNA synthesis with oligo dT primer and SuperScript RT-III (Invitrogen). Quantitative PCR was performed using Applied Biosystems 7500 Fast equipment and software. Actin transcript levels were calculated relative to reference gene Rp49. Estimates based on different PCR products indicated Actin5C mRNA levels to be 180 -fold higher than primary transcript.

\section{Under agarose invasion assay}

MB MDA-231 cells were trypsinized $24 \mathrm{~h}$ after a second siRNA treatment, split in half, and replated in DMEM $+10 \%$ FBS \pm Tet into six-well plates containing five agarose drops $(9 \mu \mathrm{L}$ of $0.5 \%$ agar $+1 \mu \mathrm{L}$ of PBS) using lowmelting-point agarose (Invitrogen). From the most central drop, phase-contrast images of the edges were taken $24 \mathrm{~h}$ later on an Olympus IX70 microscope with $5 \times$ magnification. The distance, $d$, of cell invasion was obtained by dividing the area covered by the width of the cell sheet (see Fig. 4A).

\section{Flies and genetics}

Most fly strains were from Bloomington Drosophila Stock Center. Flies were reared under standard conditions at $25^{\circ} \mathrm{C}$. For mal-d ${ }^{S 2}$ mutant clones (MARCM system) (Lee and Luo 1999), third instar larvae were heatshocked for $45 \mathrm{~min}$ at $37^{\circ} \mathrm{C}$ on three consecutive days. Injections for transgenics were performed by BestGene except tub-mal-d-3xGFP (EMBL). The AttB constructs were inserted in AttP 51D. P-element transformation (in $\mathrm{w}^{1118}$ ) was used for tub mal-d $3 \times$ GFP and HS-Act5C to get second and third chromosome viable insertions, respectively. Other fly strains used were mald $d^{\Delta 7}$ and mal- $d^{S 2}$ (Somogyi and Rørth 2004); slboGal4 (P. Rørth); and $w^{1118}, \mathrm{OR}^{+}$and EP-Act5C (Bloomington Drosophila Stock Center nos. 22549 and 20718). mal-d ${ }^{S 2}$ appears to be a null allele: The migration phenotype mimics that of an SRF-null (deletion) allele or mal- $d^{\Delta 7}$ (no detectable protein); stop codons at the same position in mammalian mrtfs generate nonfunctional, dominant-negative protein. 


\section{Analysis of ovaries in fixed samples}

Fixed-sample staining and analysis was done as described previously (Duchek et al. 2001). Images were acquired on a Zeiss confocal microscope.

The primers used and the details of subcloning and tissue culture (S2 and MB MDA-231 cells) are in the Supplemental Material.

\section{Acknowledgments}

We thank Oguz Kanca for making tub-mal-d-GFP, Valentina Migliori and Diana Low for help with ChIP and processing, Najwa Talib (BSF) for FACS sorting, Graham Wright for input on microscopy, Kiamun Woo and Isaac Kok Hwee Lim technical help, Bob Robinson for stimulating discussions about actin, and Stephen Cohen for comments on the manuscript.

\section{References}

Bershadsky AD, Gluck U, Denisenko ON, Sklyarova TV, Spector I, Ben-Ze'ev A. 1995. The state of actin assembly regulates actin and vinculin expression by a feedback loop. J Cell Sci 108: 1183-1193.

Bunnell TM, Burbach BJ, Shimizu Y, Ervasti JM. 2011. $\beta$-Actin specifically controls cell growth, migration, and the G-actin pool. Mol Biol Cell 22: 4047-4058.

Connelly JT, Gautrot JE, Trappmann B, Tan DW, Donati G, Huck WT, Watt FM. 2010. Actin and serum response factor transduce physical cues from the microenvironment to regulate epidermal stem cell fate decisions. Nat Cell Biol 12: 711-718.

Cooper SJ, Trinklein ND, Nguyen L, Myers RM. 2007. Serum response factor binding sites differ in three human cell types. Genome Res 17: 136-144.

Duchek P, Somogyi K, Jékely G, Beccari S, Rørth P. 2001. Guidance of cell migration by the Drosophila PDGF/VEGF receptor. Cell 107: 17-26.

Geisbrecht ER, Montell DJ. 2004. A role for Drosophila IAP1-mediated caspase inhibition in Rac-dependent cell migration. Cell 118: 111-125.

Huber W, von Heydebreck A, Sultmann H, Poustka A, Vingron M. 2002. Variance stabilization applied to microarray data calibration and to the quantification of differential expression. Bioinformatics 18: S96-S104.

Huttelmaier S, Zenklusen D, Lederer M, Dictenberg J, Lorenz M, Meng X, Bassell GJ, Condeelis J, Singer RH. 2005. Spatial regulation of $\beta$-actin translation by Src-dependent phosphorylation of ZBP1. Nature 438: 512-515.

Laevsky G, Knecht DA. 2001. Under-agarose folate chemotaxis of Dictyostelium discoideum amoebae in permissive and mechanically inhibited conditions. Biotechniques 31: 1140-1149.

Laevsky G, Knecht DA. 2003. Cross-linking of actin filaments by myosin II is a major contributor to cortical integrity and cell motility in restrictive environments. J Cell Sci 116: 3761-3770.

Lavoie JN, Hickey E, Weber LA, Landry J. 1993. Modulation of actin microfilament dynamics and fluid phase pinocytosis by phosphorylation of heat shock protein 27. J Biol Chem 268: 24210-24214.

Lee T, Luo L. 1999. Mosaic analysis with a repressible cell marker for studies of gene function in neuronal morphogenesis. Neuron 22: 451-461.

Luxenburg C, Pasolli HA, Williams SE, Fuchs E. 2011. Developmental roles for Srf, cortical cytoskeleton and cell shape in epidermal spindle orientation. Nat Cell Biol 13: 203-214.

Lyubimova A, Bershadsky AD, Ben-Ze'ev A. 1999. Autoregulation of actin synthesis requires the $3^{\prime}$-UTR of actin mRNA and protects cells from actin overproduction. J Cell Biochem 76: 1-12.

McGee KM, Vartiainen MK, Khaw PT, Treisman R, Bailly M. 2011. Nuclear transport of the serum response factor coactivator MRTF-A is downregulated at tensional homeostasis. EMBO Rep 12: 963-970.

Medjkane S, Perez-Sanchez C, Gaggioli C, Sahai E, Treisman R. 2009. Myocardin-related transcription factors and SRF are required for cytoskeletal dynamics and experimental metastasis. Nat Cell Biol 11: 257-268.

Miano JM, Long X, Fujiwara K. 2007. Serum response factor: master regulator of the actin cytoskeleton and contractile apparatus. Am J Physiol Cell Physiol 292: C70-C81.

Miralles F, Posern G, Zaromytidou AI, Treisman R. 2003. Actin dynamics control SRF activity by regulation of its coactivator MAL. Cell 113: 329-342.

Montagne J, Groppe J, Guillemin K, Krasnow MA, Gehring WI, Affolter M. 1996. The Drosophila serum response factor gene is required for the formation of intervein tissue of the wing and is allelic to blistered. Development 122: 2589-2597.
Mounier N, Arrigo AP. 2002. Actin cytoskeleton and small heat shock proteins: how do they interact? Cell Stress Chaperones 7: 167-176.

Negre N, Lavrov S, Hennetin J, Bellis M, Cavalli G. 2006. Mapping the distribution of chromatin proteins by ChIP on chip. Methods Enzymol 410: 316-341.

Nelson RD, Quie PG, Simmons RL. 1975. Chemotaxis under agarose: a new and simple method for measuring chemotaxis and spontaneous migration of human polymorphonuclear leukocytes and monocytes. I Immunol 115: 1650-1656.

Pinheiro EM, Xie Z, Norovich AL, Vidaki M, Tsai LH, Gertler FB. 2011. Lpd depletion reveals that SRF specifies radial versus tangential migration of pyramidal neurons. Nat Cell Biol 13: 989-995.

Pollard TD, Borisy GG. 2003. Cellular motility driven by assembly and disassembly of actin filaments. Cell 112: 453-465.

Posern G, Sotiropoulos A, Treisman R. 2002. Mutant actins demonstrate a role for unpolymerized actin in control of transcription by serum response factor. Mol Biol Cell 13: 4167-4178.

Somogyi K, Rørth P. 2004. Evidence for tension-based regulation of Drosophila MAL and SRF during invasive cell migration. Dev Cell 7: 85-93.

Sotiropoulos A, Gineitis D, Copeland J, Treisman R. 1999. Signalregulated activation of serum response factor is mediated by changes in actin dynamics. Cell 98: 159-169.

Tilney LG, Connelly P, Smith S, Guild GM. 1996. F-actin bundles in Drosophila bristles are assembled from modules composed of short filaments. J Cell Biol 135: 1291-1308.

Wagner CR, Mahowald AP, Miller KG. 2002. One of the two cytoplasmic actin isoforms in Drosophila is essential. Proc Natl Acad Sci 99: 8037-8042.

Whitmarsh AJ, Shore P, Sharrocks AD, Davis RJ. 1995. Integration of MAP kinase signal transduction pathways at the serum response element. Science 269: 403-407.

Wiggins HL, Rappoport JZ. 2010. An agarose spot assay for chemotactic invasion. Biotechniques 48: 121-124.

Yao J, Sasaki Y, Wen Z, Bassell GJ, Zheng JQ. 2006. An essential role for $\beta$-actin mRNA localization and translation in $\mathrm{Ca}^{2+}$-dependent growth cone guidance. Nat Neurosci 9: 1265-1273.

Zhang Y, Liu T, Meyer CA, Eeckhoute J, Johnson DS, Bernstein BE, Nusbaum C, Myers RM, Brown M, Li W, et al. 2008. Model-based analysis of ChIP-seq (MACS). Genome Biol 9: R137. 


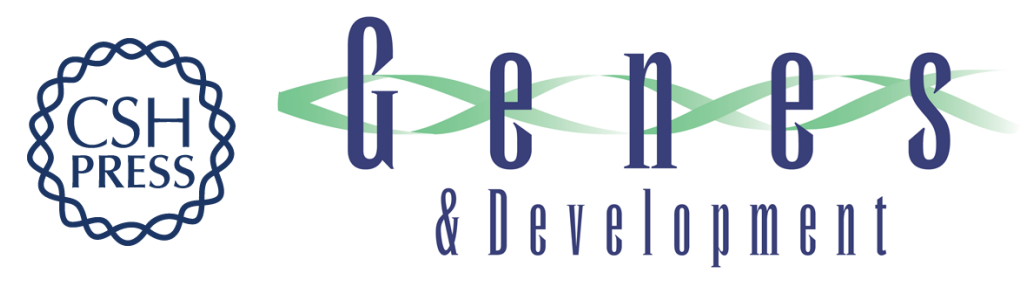

\section{The core and conserved role of MAL is homeostatic regulation of actin levels}

Lara Salvany, Julius Muller, Ernesto Guccione, et al.

Genes Dev. 2014, 28:

Access the most recent version at doi:10.1101/gad.237743.114

\section{Supplemental http://genesdev.cshlp.org/content/suppl/2014/05/14/28.10.1048.DC1 Material}

References This article cites 33 articles, 12 of which can be accessed free at: http://genesdev.cshlp.org/content/28/10/1048.full.html\#ref-list-1

Creative This article is distributed exclusively by Cold Spring Harbor Laboratory Press for the first Commons six months after the full-issue publication date (see

License http://genesdev.cshlp.org/site/misc/terms.xhtml). After six months, it is available under a Creative Commons License (Attribution-NonCommercial 4.0 International), as described at http://creativecommons.org/licenses/by-nc/4.0/.

Email Alerting Receive free email alerts when new articles cite this article - sign up in the box at the top Service right corner of the article or click here.

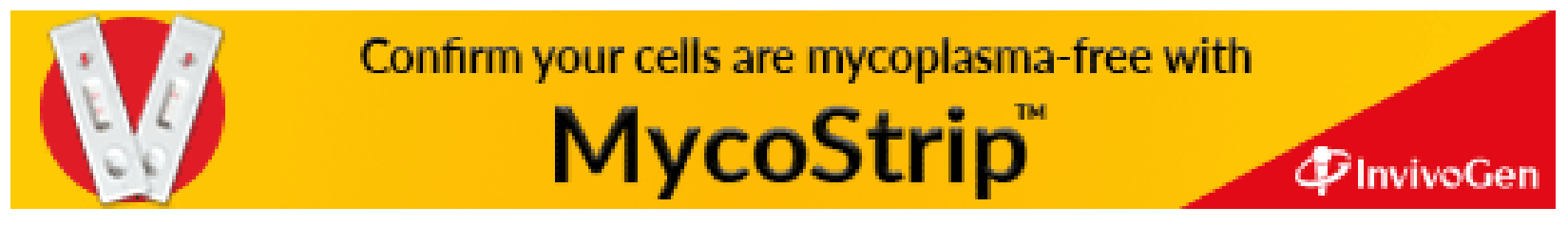

